\title{
A cell-cycle signature classifier for pan-cancer analysis
}

\author{
Neha Tabassum $\mathbb{1}^{1} \cdot$ Theodora A. Constantin $^{1} \cdot$ Biancastella Cereser $\mathbb{D}^{1} \cdot$ Justin Stebbing ${ }^{1}$
}

Received: 15 June 2020 / Revised: 25 June 2020 / Accepted: 10 August 2020 / Published online: 20 August 2020

(c) The Author(s), under exclusive licence to Springer Nature Limited 2020, corrected publication 2021

Pan-cancer genomics studies have highlighted both driver mutations and prognostic indicators. The pan-cancer atlas initiative compares 32 tumour types profiled by The Cancer Genome Atlas (TCGA) and comprises DNA and RNA profiles derived from 11,069 human tumours. Integration of these datasets allows a comprehensive and some might say holistic analysis of the molecular aberrations and functional consequences across these types. It also allows association studies between the frequency of DNA alterations and activity scores of pathways with fundamental biological role of cancer. The cell-cycle pathways represent a compelling choice for such pathway activity-based analysis [1,2], and the cell-cycle score signature (CCS) applied by Lundberg et al. here has been previously validated by the same research group in a number of breast cancer samples [3, 4]. In this issue of journal, Lundberg et al. hypothesised that a correlation between cell-cycle pathways and genomic features of a tumour can be made for a variety of malignancies, which could significantly expand the clinical application of the methodology in the provision of independent prognostic information.

The previously invented CCS by the authors had cut-offs specific to the breast cancer, so the authors modified the existing CCS system for the current analysis, by creating new tertiles on the basis of the continuous CCS in the pancancer dataset alone. This novel CCS score is generated by methodology utilising 463 genes that have previously been identified to have implications on general cell-cycle activity based on the amalgamation of three different databasesKEGG, HNGC and Cyclebase 3.0 [3, 4]. The CCS score was then used for each individual tumour type in the TCGA pan-cancer dataset to generate low, intermediate and high

These authors contributed equally: Neha Tabassum, Theodora A. Constantin

Neha Tabassum

n.tabassum16@imperial.ac.uk

1 Department of Surgery and Cancer, Imperial College, London, UK
CCS subgroups. In doing so, Lundberg et al. have generated an impressive amount of correlation data, which could be useful for both the general understanding of cancer biology and tumour classification. Higher CCS scores are characteristics of tumours with advanced pathological stage and subjected to radiotherapy. The latter is not surprising, if we consider how the activation of cell-cycle checkpoints and how DNA-repair proteins, such as p53, play a role in the physiological response to ionizing radiation [5]. A confirmation of this is also the correlation between the number of mutations in TP53 and CCS activity, which the authors could determine with their novel approach.

The regulation of cell-cycle progression by PIK3CA has been highlighted in the recent years [6], making the protein a potential therapeutic target for cancer treatment. This evidence is also confirmed by the study from Lundberg et al., where, similarly to TP53, mutations in PIK3CA are also more frequent in cancers with a high CCS score. On the other hand, mutations in BRAF were not associated with higher CCS scores. This may indicate that regulation of cellcycle molecules by this oncogene may not be direct as with p53 or PIK3CA, but it exerts its oncogenic role in different ways or may require a partner for cell-cycle regulation.

These data, together with the identification of the chromosomal arms associated with CCS-correlated aneuploidy, highlights the potential of the CCS methodology. We do not currently know the impact of every gene in the cell cycle, and the role of less frequently mutated or expressed genes is elusive. By analysing the dataset used here, it would be possible to infer the contribution of any gene of interest on cell-cycle analysis, to then validate it in vitro in either cancer or other systems.

While the classifier successfully groups tumours in these categories, it also highlights once again how heterogenous cancer types are. Using different algorithms, the authors confirmed that cell-cycle signatures vary among different tumours. Lack of extensive single-cell signature data does not permit application of the CCS score to measure intratumor heterogeneity, but this is not considered a limitation of this current study. When more extensive application of 
single-cell analysis will be available, the tool by Lundberg et al. could provide insights of how cell-cycle signatures are among the most heterogeneous tumours. It could ultimately provide prospective information regarding the responses of subsets of cancers to treatments, including radiotherapy. The most ambitious scope of the study was to assess the relationship between cell-cycle activity and clinical outcome, in particular progression free interval. The use of a CCS classifier provided significant prognostic information for four tumour types: renal cell carcinoma, low grade glioma, sarcoma and uveal melanoma. In addition, CCS tertiles derived at a pan-cancer level provided more prognostic information than CCS tertiles derived at an intracancer level. This in turn suggests that deriving cut-off points for transcriptional biomarkers on a pan-cancer level, instead of deriving them in a single cancer type, may be advantageous, although this requires validation.

The CCS is, however, not without limitations: the tumour proliferation rate is not the only factor that determines prognosis. Other factors include increased susceptibility in acquiring genetic alterations or age [7]. For example, follicular lymphomas are indolent in nature, but they often are associated with a poor prognosis [8]. Certain limitations also arise from utilising the TCGA dataset. First, as the authors mentioned that the analysis includes data from DNA and RNA levels, but lacks protein and methylation array data. Second, in the absence of single-cell resolution data, the CCS is derived from average gene signals across an entire sample and therefore does not take tumour heterogeneity into account. As the results are generalised it compromises the claim that certain mutations are more prevalent in all 'aggressive phenotypes'. Third, external validation for the CCS signature was not possible as TCGA is the only dataset available that provides pancancer data, although by using a pan-cancer approach, the hope is that the CCS can be applied as a general tool to examine individual tumours.

In aggregate, this study provides useful information on the frequency of DNA aberrations found in tumours classified based on the cell-cycle activity and demonstrates the prognostic ability of cell-cycle gene expression score derived at a pan-cancer level. It would be of interest to see how such tool can serve as an additional independent assessment of prognosis in specific cancers. However, the data, as it currently stands unvalidated, cannot be applied solely to specific cancers and this limits its use in a clinical context, while showing a higher level of applicability in the more generic field of cancer biology.

\section{Compliance with ethical standards}

Conflict of interest JS and BC reports his conflicts at https://www.na ture.com/onc/editors. JS and BC had no role in the review process.

Publisher's note Springer Nature remains neutral with regard to jurisdictional claims in published maps and institutional affiliations.

\section{References}

1. Hartwell LH, Kastan MB. Cell cycle control and cancer. Science. 1994;266:1821-8.

2. Malumbres M, Barbacid M. Cell cycle, CDKs and cancer: a changing paradigm. Nat Rev Cancer. 2009;9:153-66.

3. Lundberg A, Lindström LS, Harrell JC, Falato C, Carlson JW, Wright PK, et al. Gene expression signatures and immunohistochemical subtypes add prognostic value to each other in breast cancer cohorts. Clin Cancer Res. 2017;23:7512-20.

4. Tobin NP, Lundberg A, Lindström LS, Harrell JC, Foukakis T, Carlsson $\mathrm{L}$, et al. PAM50 provides prognostic information when applied to the lymph node metastases of advanced breast cancer patients. Clin Cancer Res. 2017;23:7225-31.

5. Pawlik TM, Keyomarsi K. Role of cell cycle in mediating sensitivity to radiotherapy. Int $\mathrm{J}$ Radiat Oncol Biol Phys. 2004;59:928-42.

6. Chang F, Lee JT, Navolanic PM, Steelman LS, Shelton JG, Blalock $\mathrm{WL}$, et al. Involvement of PI3K/Akt pathway in cell cycle progression, apoptosis, and neoplastic transformation: a target for cancer chemotherapy. Leukemia. 2003;17:590-603.

7. Gospodarowicz M, Mackillop W, O'Sullivan B, Sobin L, Henson D, Hutter RV, et al. Prognostic factors in clinical decision making: the future. Cancer. 2001;91:1688-95.

8. Mozas P, Nadeu F, Rivas-Delgado A, Rivero A, Garrote M, Balagué $\mathrm{O}$, et al. Patterns of change in treatment, response, and outcome in patients with follicular lymphoma over the last four decades: a single-center experience. Blood Cancer J. 2020;10:31. 